\title{
Detecting the Inverted-U in fMRI Studies of Schizophrenia: A Comparison of Three Analysis Methods
}

\author{
Michael L. Thomas ${ }^{1, *}$ (D) , John R. Duffy ${ }^{1}$, Neal Swerdlow ${ }^{2}$, Gregory A. Light ${ }^{2}$ and Gregory G. Brown ${ }^{2}$ (D) \\ ${ }^{1}$ Department of Psychology, Colorado State University, Fort Collins, CO, USA \\ ${ }^{2}$ Department of Psychiatry, University of California San Diego, La Jolla, CA, USA \\ (Received November 21, 2020; Final revision February 23, 2021; Accepted March 12, 2021; First Published Online May 5, 2021)
}

\begin{abstract}
Objective: Cognitive tasks are used to probe neuronal activity during functional magnetic resonance imaging (fMRI) to detect signs of aberrant cognitive functioning in patients diagnosed with schizophrenia (SZ). However, nonlinear (inverted-U-shaped) associations between neuronal activity and task difficulty can lead to misinterpretation of group differences between patients and healthy comparison subjects (HCs). In this paper, we evaluated a novel method for correcting these misinterpretations based on conditional performance analysis. Method: Participants included $25 \mathrm{HCs}$ and 27 SZs who performed a working memory (WM) task (N-back) with 5 load conditions while undergoing fMRI. Neuronal activity was regressed onto: 1) task load (i.e., parametric task levels), 2) marginal task performance (i.e., performance averaged over all load conditions), or 3) conditional task performance (i.e., performance within each load condition). Results: In most regions of interest, conditional performance analysis uniquely revealed inverted-U-shaped neuronal activity in both SZs and HCs. After accounting for conditional performance differences between groups, we observed few difference in both the pattern and level of neuronal activity between SZs and HCs within regions that are classically associated with WM functioning (e.g., posterior dorsolateral prefrontal and parietal association cortices). However, SZs did show aberrant activity within the anterior dorsolateral prefrontal cortex. Conclusions: Interpretations of differences in neuronal activity between groups, and of associations between neuronal activity and performance, should be considered within the context of task performance. Whether conditional performance-based differences reflect compensation, dedifferentiation, or other processes is not a question that is easily resolved by examining activation and performance data alone.
\end{abstract}

Keywords: Neuroimaging, Schizophrenia, Psychometrics, Working Memory, Confounds

\section{INTRODUCTION}

Cognitive tasks are used during functional magnetic resonance imaging (fMRI) to probe for signs of aberrant neuronal functioning in patients diagnosed with schizophrenia (SZs). However, since brain responses vary with task performance, and because performance differs between patients and healthy control subjects (HCs), it is often unclear whether different patterns of neuronal response between groups should be attributed to intrinsic differences in brain function, differences in performance, or both (Brown \& Eyler, 2006; Callicott et al., 2003; Manoach, 2003).

Typically, one of two approaches is used to understand the relationship between performance and neuronal activity

*Correspondence concerning this article should be addressed to Michael Thomas, Department of Psychology, Colorado State University, Fort Collins, CO, USA. E-mail: Michael.L.Thomas@colostate.edu differences between groups. The first approach is to examine neuronal activity as a function of task difficulty (e.g., Cohen et al., 1997; Jansma, 2004). This approach is able to characterize different patterns of activity across task difficulty levels but obscures the impact of group performance levels on brain activation. The second approach is to examine neuronal activity as a function of marginal (or average) task performance levels (e.g., Karlsgodt et al., 2009; Manoach et al., 1999). This approach is able to characterize patterns of activity across task performance levels but obscures the impact of task difficulty levels on neuronal activity. Consequently, neither of these approaches examines differences in both the pattern and level of neuronal activity between patient and control groups. We aimed to resolve this problem using a novel methodology: conditional performance analysis. That is, the examination of neuronal activity as a function of performance within specific difficulty levels. 


\section{Neuroimaging of Working Memory in Schizophrenia}

Working memory (WM) is defined as the “... mechanisms or processes that are involved in the control, regulation and active maintenance of task-relevant information in the service of complex cognition..." (Miyake \& Shah, 1999, p. 450). WM deficits are a core feature of schizophrenia (Barch \& Smith, 2008; Kalkstein, Hurford, \& Gur, 2010; Lee \& Park, 2005), as well as a predictor of poor functional outcome (Green, Kern, Braff, \& Mintz, 2000). In the attempt to better understand WM deficits, researchers often rely on neuroimaging methods, especially fMRI (Brown \& Eyler, 2006).

Unfortunately, inconsistencies in the properties of tasks used can complicate interpretations of brain imaging results (Gur, Erwin, \& Gur, 1992). This is due, in part, to nonlinear neuronal response functions. Early fMRI research demonstrated that increased task difficulty results in increased brain response in cortical regions thought to be involved in WM (Desmond, Gabrieli, Wagner, Ginier, \& Glover, 1997; Manoach et al., 1997). However, an early PET imaging study by Goldberg and colleagues suggested that neuronal activity is not monotonic, but rather can also decrease as demands become excessive (1998). More specifically, Callicott and colleagues (1999) found an inverted-U-shaped response pattern in the dorsolateral prefrontal cortex (DLPFC). Presumably, task disengagement- the descending slope of the inverted-U-is related to high computational costs (Westbrook \& Braver, 2015); that is, if the examinee deems that cognitive effort is unlikely to result in accurate performance (and reward), they might disengage resources.

Studies comparing the DLPFC response between patients and controls suggest that nonlinearity can confuse the comparison of neuronal activity between groups. Specifically, because patients are assumed to have "left-shifted" inverted-U-shaped neuronal response functions-that is, the neuronal response peaks at lower levels of difficultyusing tasks with varying levels of difficulty between studies can produce contradictory patterns. For example, Glahn et al. conducted a meta-analysis of fMRI research using a popular WM task ( $N$-back) and concluded that SZs demonstrate hypoactivation in the DLPFC (Glahn et al., 2005). However, this conclusion was challenged by a significant minority of studies finding hyperactivation (Brown \& Thompson, 2010). Presumably, evidence that SZs show less neuronal activity during task engagement is an artifact that reflects examinees' experience of being overwhelmed by task difficulty. Manoach and colleagues, for example, suggested that, because “... DLPFC activation may reflect WM demand only up to the point at which the demands begin to outstrip WM capacity" (Manoach et al., 1999, p. 1135), combined with the fact that patients demonstrate worse performance, “. . . subjects may [engage in] cognitive and affective processes that [are] unrelated to WM [such as] error monitoring, attempts at compensation, disengaging from the task, feeling overwhelmed, and guessing" (Manoach et al., 1999, p. 1129).
Studies have provided mixed support for the inverted-U model of neuronal activity in both HCs and SZs, particularly as they relate to the theory that, after accounting for the "leftshift," patients demonstrate inefficient (i.e., greater) activity (Callicott et al., 2000; Jansma, 2004; Karlsgodt et al., 2009; Manoach et al., 2000; Potkin et al., 2009; Van Snellenberg et al., 2016; Zou, Gu, Wang, Gao, \& Yang, 2011). The methodological challenge is clear: How should differences in neuronal activity be interpreted between patient and control groups given that task performance also differs? Some researchers focus on fMRI data produced by items or tasks where performance does not differ between groups. This might include selecting subsets of individuals from each group that are matched on performance, parametrically manipulating task conditions on an individual basis, or evaluating only correct trials in event-related designs (Brown \& Eyler, 2006; Jansma, 2004; Potkin et al., 2009). However, matching performance between groups can artificially create a scenario that does not reflect the original abnormality, thus creating ambiguity when interpreting neurophysiological results (Zhou et al., 2016). More broadly, this problem is known as the performance-activation dilemma (Brown \& Eyler, 2006) and poses a significant obstacle to the development of comprehensive, brain-cognitive models of mental illness.

\section{The Inverted-U: Sources of Misinterpretation}

Examining neuronal activity differences between patient and control groups without considering group differences in the match between ability and task difficulty can be counterproductive. To help explain why, Figure 1 presents several possible scenarios based on the inverted- $U$ model of neuronal activity. To simplify the analysis, the model assumes that the relationship between neuronal activity and task difficulty is a normal distribution with equal group variances and with the mode of the schizophrenia group "left-shifted" toward less difficult task conditions compared to controls. Note that in both groups, it is assumed that activity increases with difficulty up to some critical point and then decreases. Not all tasks produce inverted-U-shaped activity; thus, here we are limiting our discussion to tasks where the relationship between difficulty and brain activation follows this theoretical model.

Figure 1 plots neuronal activity as a function of three levels of task difficulty (non-shaded region of each plot): a low difficulty task, a moderate difficulty task, and a high difficulty task. The left panel shows the pattern of hyperactivation among SZs when overall task difficulty is low. In this scenario, the SZs are expected to show greater neuronal activity than HCs, because, whereas SZs are moderately challenged by the task, HCs are only very weakly challenged. In other words, left panel is indicative of a task that is too easy from HCs but appropriately difficult for SZs. The middle panel shows the pattern of no difference in activity between SZs and HCs when overall task difficulty is moderate. SZs are 
- Healthy comparison subjects (HCs)

- Schizophrenia patients (SZs)
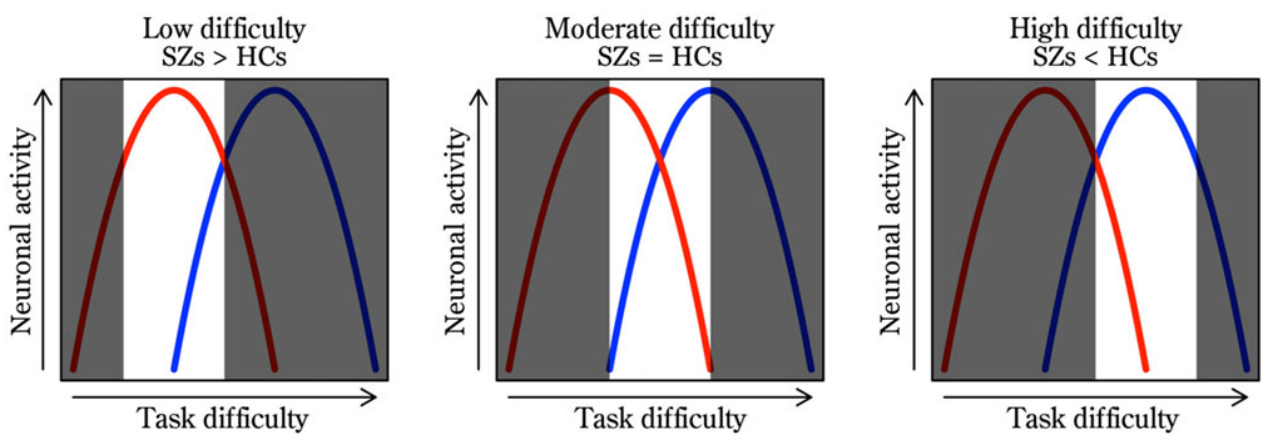

Fig. 1. Conceptual inverted-U models of neuronal activity as a function of task difficulty assuming a left-shift of the neuronal activity-task difficulty curve in the SZ group. Differences in difficulty are show as a right-shift of the unshaded window. Left panel: low difficulty tasks are expected to result in hyperactivation among SZs, as indicated by the elevation of the SZ curve over the HC curve within the difficulty window. Middle panel: moderate difficulty tasks are expected to result in little difference in neuronal activity between groups. Right panel: high difficulty tasks are expected to result in hypoactivation among SZs. Bottom-left panel: low difficulty tasks are expected to result in no association between performance and neuronal activity among SZs, but a negative association among HCs.

highly challenged and HCs are beginning to be moderately challenged. However, because mirror sides of the inverted$\mathrm{U}$ functions are engaged, the expected result is no overall group differences in neuronal activity. Finally, the right panel shows the pattern of hypoactivation among SZs-which is most often reported in the literature - that occurs when overall task difficulty is high. The task in this scenario is too hard for SZs but appropriately difficult for HCs.

In this paper, we test the assumption that examining neuronal activity as a function of performance conditioned on task difficulty (i.e., WM load) will resolve these interpretative challenges. Conditional performance analysis requires the investigator to estimate individual subjects' performance (e.g., accuracy) at each level of task difficulty (e.g., each level of WM load) and then to analyze the data as a repeated measures design. The key argument for using this approach is that neuronal activity should be examined as a function of conditional performance and not task difficult or even marginal (i.e., average) performance.

\section{Study Aims}

Our study had three aims. The first aim was hypothesisdriven and the second and third aims were exploratory. The first aim was to demonstrate the use of conditional performance analysis for examining and comparing loadresponse curves between groups of patients and controls. We hypothesized that conditional performance analysis would uniquely and consistently reveal nonlinear (invertedU) shaped neuronal activity in both groups. The second aim was to compare brain response between SZs and HCs in order to explore the neural inefficiency model of WM deficits in schizophrenia. The third aim was to explore demographic, clinical, and cognitive correlates of inefficient neuronal activity. We also conducted whole-brain, voxel- wise exploratory analyses of group and group-by-conditional performance effects.

\section{METHODS}

\section{Participants}

Data were collected from 27 SZs (schizophrenia or schizoaffective disorder) and $25 \mathrm{HCs}$. We aimed to recruit male and female adults aged 18-70 years with adequate hearing and eyesight who were fluent in English and able to perform the cognitive and imaging tasks required. Exclusion criteria were inability to understand or give consent, positive drug toxicology screen (except marijuana), alcohol or substance dependence in the preceding 6 months, pregnancy, contraindications and conditions incompatible with MRI, previous significant head injury, significant extrapyramidal symptoms or tardive dyskinesia, and significant medical or neurological diagnoses (except diabetes). Controls were additionally excluded if they were enrolled in special education courses during school or met diagnostic criteria for any psychosis spectrum disorder or bipolar disorder. Clinical diagnoses were verified using a structured clinical interview administered by a licensed psychologist (First, Williams, Karg, \& Spitzer, 2015). We did not exclude left-handed participants because left-handedness tends to be higher among people with psychosis (Webb et al., 2013), and thus we did not want to limit the generalizability of our results. The final proportion of left-handed individuals was nearly identical between HCs $(3 / 25 ; 12 \%)$ and SZs (4/27; 15\%), and Fisher's exact test indicated no significant association between handedness and group. Written consent was obtained from all participants. Research procedures were reviewed and approved by the UC San Diego Institutional Review Board. 


\section{Experimental and Behavioral Task Design}

The experimental design was a two-by-five mixed factorial. The between-subjects factor was population (i.e., HC vs. SZ; two levels). The within-subjects factor was WM load (five levels). To manipulate load, participants were administered the N-back task. The N-back task is a forced-choice measure of WM that requires examinees to monitor a continuous stream of stimuli (pseudowords) and respond each time an item is repeated from $N$ before. This task was chosen because it is commonly used to study brain and cognitive functioning in SZs (Glahn et al., 2005). We created three N-back runs, each consisting of five blocks of trials (i.e., 1- through 5-back load conditions). One run was administered outside of the scanner and the remaining two were administered within the scanner. Blocks were counterbalanced over runs. The task was administered using PsychoPy (Peirce et al., 2019). Pseudowords were presented in white font on a black background for $2500 \mathrm{~ms}$ with a $500 \mathrm{~ms}$ inter-item interval. The timing was constrained so that each block would last exactly $60 \mathrm{~s}$. Blocks were separated by $20 \mathrm{~s}$ intervals (with a fixation cross). Additional experimental and task design details are provided in the Supplemental Materials.

\section{Clinical and Cognitive Measures}

Symptoms were assessed using the Scale for the Assessment of Negative Symptoms (SANS) and Scale for the Assessment of Positive Symptoms (SAPS) (Andreasen, 1984b, 1984a). Cognitive measures included Trail Making Test: Part A (TMT), Brief Assessment of Cognition in Schizophrenia Symbol Coding (BASC), Hopkins Verbal Learning Test (HVLT), Letter-Number Sequencing (LNS), and Category (Animal) Fluency from the NIMH MATRICS Consensus Cognitive Battery (MCCB) (Green et al., 2004). We also administered the Wide Range Achievement Test 3rd Edition (WRAT-3) Reading subset. Effort was objectively measured using the Rey 15-Item task with the extended recognition trial (Boone, Salazar, Lu, Warner-Chacon, \& Razani, 2002) and subjectively measured using the NASA Task Load Index (Hart \& Staveland, 1988). Functioning was measured using the Role Functioning Scale (Goodman, Sewell, Cooley, \& Leavitt, 1993).

\section{Image Acquisition}

Participants were scanned using a General Electric (GE) Discovery MR750 3.0 Tesla whole-body imaging system and a Nova 32-channel head coil. Anatomical scans were based on a T1-weighted spoiled gradient echo sequence with fast and prospective motion correction imaging options $\left(\mathrm{TR}=7.4 \mathrm{~ms} ; \mathrm{TI}=1060 \mathrm{~ms} ; \mathrm{TE}=2.3 \mathrm{~ms} ;\right.$ flip angle $=8^{\circ}$; FOV $=25.6 \mathrm{~cm}$; matrix size $=320 \times 320$; in-plane resolution $=.8 \mathrm{~mm}$; slice thickness $=.8 \mathrm{~mm}$; slices $=204$; slice spacing $=0$ ) acquired parallel to the sagittal plane in an interleaved manner. Functional scans sensitive to the T2-weighted blood-oxygen-level-dependent (BOLD) signal were collected using a gradient echo pulse sequence with multiband and echo-planar imaging options $(\mathrm{TR}=800 \mathrm{~ms}$; $\mathrm{TE}=25 \mathrm{~ms}$; flip angle $=52^{\circ} ; \mathrm{FOV}=20.8 \mathrm{~cm}$; matrix size $=86 \times 86$; in-plane resolution $=2.42 \mathrm{~mm}$; slice thickness $=2.4 \mathrm{~mm}$; slices $=10$ [60 effective]; slice spacing $=0$; multiband factor $=6$ ) acquired parallel to the intercommissural (AC-PC) plane in an interleaved manner.

\section{Image Processing and Regions of Interest (ROIs)}

We used software from Analysis of Functional NeuroImages (AFNI; Ver. 18.1.14) (Cox, 1996) and FMRIB Software Library (FSL; Ver. 5.0.10) (Jenkinson, Beckmann, Behrens, Woolrich, \& Smith, 2012) to preprocess the structural and functional images. A detailed description of preprocessing steps is included in the Supplemental Materials. Briefly, images were processed using a pipeline that included segmentation, distortion correct, despiking, alignment and co-registration of the functional images to the structural images, detection of outliers, and blurring. To account for physiological motion, respiration and cardiac activity were acquired in parallel with the functional images and converted to sines and cosines of the first- and second-phase cycles modeling of the physiological activity (Glover, $\mathrm{Li}, \&$ Ress, 2000). Using AFNI's 3dDeconvolve tool, a general linear model (GLM) was then applied to each participant's co-registered functional images and movement time series data (ignoring censored values). The GLM analysis incorporated covariates accounting for linear, quadratic, cubic, and quartic drift, six motion parameters, eight physiological noise regressors, and the reference functions. The reference functions were vectors representing the behavioral paradigm convolved with a model of the hemodynamic response using a gamma function.

To prepare for group analyses, individual statistical maps reflecting parameter estimates for each simple contrast (1-, 2-, 3-, 4-, and 5-back vs. low-level fixation baseline) were rescaled to reflect percent signal change. We then removed non-brain tissue from the functional maps using AFNI's 3dresample tool with masks based on the structural images. AFNI's adwarp tool was then used to warp the masked individual statistical maps into Talairach space using the ICBM452 brain template (Rex, Ma, \& Toga, 2003).

Regions of interest (ROIs) were based on results from a quantitative meta-analysis on the N-back task using the activation likelihood estimates (Owen, McMillan, Laird, \& Bullmore, 2005). Specifically, using the meta-analysis coordinates, we selected ROIs in regions traditionally considered to be part of the DLPFC, ventrolateral prefrontal cortex (VLPFC), parietal association cortex (PAC), and dorsal midcingulate cortex (dMCC). The first two ROIs were in the DLPFC. The first was centered near the anterior portion of the left middle frontal gyrus (aMFG) primarily near Brodmann area 10 (RAI x $=38, \mathrm{y}=-44, \mathrm{z}=20$; radius $=$ $6 \mathrm{~mm}$ ) and the second was centered near the posterior portion of the left middle frontal gyrus (pMFG) primarily near 
Brodmann area 46 (RAI $\mathrm{x}=44, \mathrm{y}=-18, \mathrm{z}=22$; radius $=14.2 \mathrm{~mm}$ ). The third ROI, a marker of the VLPFC, was located near the opercular part of the inferior frontal gyrus (IFG) primarily near Brodmann area 44 (RAI $x=50$, $\mathrm{y}=-12, \mathrm{z}=5$; radius $=11.3 \mathrm{~mm}$ ). The fourth ROI was centered near the dMCC primarily near Brodmann area 32 (RAI $\mathrm{x}=2, \mathrm{y}=-12, \mathrm{z}=42$; radius $=9 \mathrm{~mm}$ ). The fifth ROI, a marker of the PAC, was centered near the left inferior parietal lobule (IPL) - mostly the supramarginal gyrus-primarily near Brodmann area 40 (RAI $\mathrm{x}=34, \mathrm{y}=48, \mathrm{z}=38$; radius $=10.7 \mathrm{~mm}$ ).

\section{Analyses}

Activation differences between groups in the ROIs were analyzed using linear mixed-effect models (Hox, 2010) within the R "Ime4" package (Bates, Mächler, Bolker, \& Walker, 2015). We regressed percent signal change in the BOLD response within each ROI onto the fixed effects of group, a single type of load performance regressor, and the interaction of group by load performance. Group was contrast-coded. Performance was operationalized as discriminability $\left(d^{\prime}\right)$ from an equal variance signal detection-item response model (Thomas et al., 2018). $d^{\prime}$ is an index of performance accuracy, but one that has been adjusted for response bias. The load performance regressor was either: (1) N-back load: 1-5; (2) marginal performance: $d^{\prime}$ averaged across load; or (3) conditional performance: $d^{\prime}$ calculated for each load level. In other words, whereas the N-back load regressor only varied over conditions (e.g., 1-back), the marginal performance regressor varied over subjects (e.g., Subject 1's d', Subject 2's $d^{\prime}$, etc.), and the conditional performance regressor varied over subjects within conditions (e.g., Subject 1's $d^{\prime}$ at 1-back, Subject 2's $d^{\prime}$ at 1-back, Subject 1's $d^{\prime}$ at 2-back, Subject 2's $d^{\prime}$ at 2-back, etc.). In all cases, the load performance regressor was coded using orthogonal linear contrasts. Random intercepts and slopes for run and order effects were included in all models. To determine whether nonlinear models fitted the data better than linear models, we compared the overall fit of models with linear or both linear and quadratic terms using the Akaike information criterion (AIC; smaller is better) and chi-square difference tests $\left(\Delta \chi^{2}\right)$. Significance values were adjusted for multiple comparisons using the false discovery rate correction (Benjamini \& Hochberg, 1995).

Separate from our ROI analyses, exploratory whole-brain, voxel-wise analyses were performed using the AFNI equitable thresholding and clustering (ETAC) technique to limit the potential false-positive rate to 5\% (Cox, 2019). ETAC was implemented as part of the $3 \mathrm{dttest}+$ tool with clustering based on voxels touching at faces or edges, two-sided $t$-tests, $p$ values of $.05, .01$, and .001 with no added blur. We conducted both one-sample and independent two-sample $t$-tests. Separate analyses were conducted with the dependent variable defined either as the average BOLD response or as the quadratic BOLD response predicted from conditional performance.
Table 1. Demographic and clinical characteristics

\begin{tabular}{lccc}
\hline \hline & $\begin{array}{c}\text { Healthy con- } \\
\text { trols (HCs) }\end{array}$ & $\begin{array}{c}\text { Schizophrenia } \\
\text { patients (SZs) }\end{array}$ & $\begin{array}{c}p \text { HCs } \\
\text { vs. SZs }\end{array}$ \\
\hline Sample size & 25 & 27 & - \\
Age & $40.60(9.38)$ & $43.26(9.59)$ & .32 \\
Age range & $23-54$ & $21-58$ & - \\
Sex: male & $16(64 \%)$ & $18(67 \%)$ & $>.999$ \\
Hispanic & $5(20 \%)$ & $8(30 \%)$ & .53 \\
Race & - & - & .37 \\
American Indian/ & $0(0 \%)$ & $1(4 \%)$ & - \\
Alaskan native & & $3(11 \%)$ & - \\
Asian & $4(16 \%)$ & $6(22 \%)$ & - \\
Black or African & $2(8 \%)$ & $2(7 \%)$ & - \\
$\quad$ American & & & \\
More than one & $5(20 \%)$ & $15(56 \%)$ & - \\
race & & $12.70(2.15)$ & $<.001$ \\
White & $14(56 \%)$ & $12.67(3.25)$ & .19 \\
Education & $15.80(2.06)$ & .01 \\
Parents' education & $13.92(3.56)$ & & \\
WRAT reading & 104.36 & $96.00(10.87)$ & - \\
$\quad$ score & $(10.98)$ & & - \\
Chlorpromazine & - & $482.61(462.83)$ & - \\
$\quad$ equivalent doses & & $21.48(9.90)$ & - \\
SAPS & - & $6.59(4.11)$ & - \\
SANS & - & & \\
\hline \hline
\end{tabular}

Note: Means and standard deviations are reported for continuous variables. Counts and percentages are reported for discrete variables. Groups were compared using regression for continuous variables and Fisher's exact test for categorical variables. Education is in years completed. SANS $=$ Scale for the Assessment of Negative Symptoms reported as total global rating scores; SAPS $=$ Scale for the Assessment of Positive Symptoms reported as total global rating scores; WRAT $=$ Wide Range Achievement Test.

\section{RESULTS}

Table 1 reports demographic and clinical characteristics of both samples. SZs and HCs differed significantly on years of education and WRAT scores, but not on age, gender, ethnicity, race, or years of parents' education. In terms of N-back task accuracy, SZs performed significantly worse overall $(t(50)=-3.586, p=.001$, Cohen's $d=-1.00)$, as well at all levels of N-back load, with Cohen's $d$ s of $-.89,-.63$, $-.58,-.53$, and -.48 for 1- through 5-back, respectively. Additionally, a nonparametric (Kolmogorov-Smirnov) test of equality between raw accuracy distributions for SZs and HCs indicated a significant difference $(D=.47, p=.002)$. Estimates of memory discriminability $\left(d^{\prime}\right)$ and bias $\left(C_{c}\right)$ over $\mathrm{N}$-back load by group are plotted in Figure 2 .

\section{Aim 1: Nonlinear Neuronal Activity}

Comparisons of models that included linear versus nonlinear (both linear and quadratic) terms are reported in Table 2. Predictions based on the best-fitting model for each ROI are reported in Figure 3. Corresponding estimates of regression parameters and effect sizes with confidence intervals are reported in Supplemental Table 1. 
Table 2. Differences in linear versus nonlinear model fit for the regression of neuronal activity onto N-back, marginal performance, and conditional performance

\begin{tabular}{|c|c|c|c|c|c|c|}
\hline ROI & Predictor & Linear AIC & Nonlinear AIC & $\Delta \chi^{2}$ & $d f$ & $p_{\mathrm{FDR}}$ \\
\hline Left aMFG & N-back & 409.56 & 413.50 & .07 & 2 & .968 \\
\hline Left aMFG & Marginal performance & 409.18 & 411.66 & 1.53 & 2 & .583 \\
\hline Left aMFG & Conditional performance & 413.40 & 403.29 & 14.11 & 2 & .004 \\
\hline Left pMFG & N-back & 132.20 & 129.21 & 6.99 & 2 & .076 \\
\hline Left pMFG & Marginal performance & 122.24 & 124.42 & 1.82 & 2 & .556 \\
\hline Left pMFG & Conditional performance & 133.35 & 126.28 & 11.07 & 2 & .015 \\
\hline Left IFG & N-back & 414.01 & 417.30 & .71 & 2 & .751 \\
\hline Left IFG & Marginal performance & 401.93 & 399.79 & 6.13 & 2 & .100 \\
\hline Left IFG & Conditional performance & 413.05 & 414.45 & 2.60 & 2 & .495 \\
\hline $\mathrm{dMCC}$ & N-back & 329.57 & 332.27 & 1.30 & 2 & .602 \\
\hline $\mathrm{dMCC}$ & Marginal performance & 320.50 & 322.70 & 1.79 & 2 & .556 \\
\hline dMCC & Conditional performance & 329.60 & 323.75 & 9.85 & 2 & .022 \\
\hline Left IPL & N-back & 121.73 & 107.90 & 17.82 & 2 & .002 \\
\hline Left IPL & Marginal performance & 111.70 & 113.27 & 2.43 & 2 & .495 \\
\hline Left IPL & Conditional performance & 113.16 & 101.14 & 16.02 & 2 & .002 \\
\hline
\end{tabular}

Note aMFG = anterior middle frontal gyrus; $\mathrm{pMFG}=$ posterior middle frontal gyrus; $\mathrm{IFG}=$ inferior frontal gyrus; $\mathrm{dMCC}=$ dorsal mid cingulate cortex; $\mathrm{IPL}=$ inferior parietal lobule; $\mathrm{AIC}=$ Akaike information criterion; $p_{\mathrm{FDR}}=$ false discovery rate corrected $\mathrm{p}$-value; $\mathrm{ROI}=$ region of interest. Statistically significant effects are highlighted in bold font.
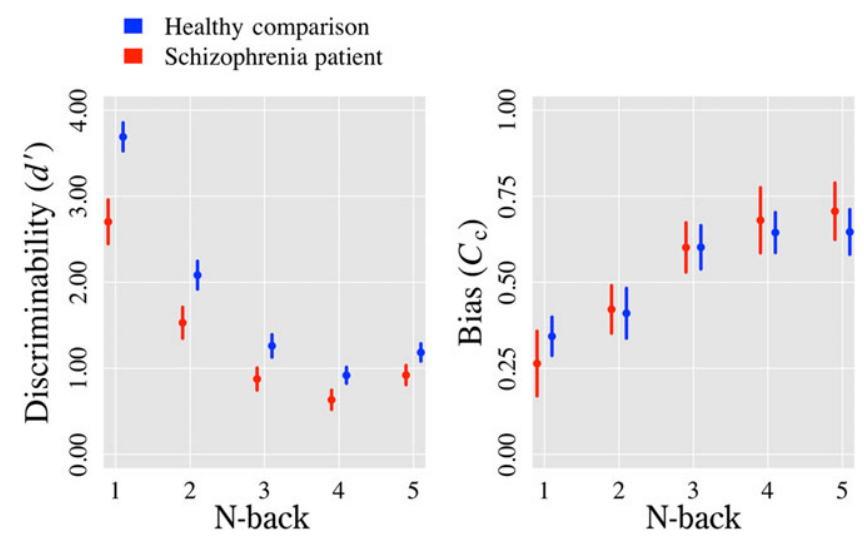

Fig. 2. Estimates of memory discriminability $\left(d^{\prime}\right)$ and bias $\left(C_{c}\right)$ over $\mathrm{N}$-back load by group. Vertical lines indicate standard errors of the means.

For the prediction of neuronal activity from N-back load, nonlinear activity was only found in the left IPL. All other comparisons indicated linear patterns of activity. In the left IPL, the patterns of activity were mixed for N-back load, with $\mathrm{HCs}$, in blue, showing a negative quadratic trend (i.e., inverted-U-shaped), and SZs, in red, showing a positive quadratic trend (i.e., U-shaped). For the prediction of neuronal activity from marginal performance, none of the nonlinear models fitted the data significantly better than models that only included linear terms. As reported in Figure 3, neuronal activity was positively associated with marginal performance for SZs and showed a mixed pattern of positive and negative trends for HCs. For the prediction of neuronal activity from conditional performance, all but one ROI favored the nonlinear model. The one exception was the left IFG. For all other
ROIs, Figure 3 shows clear evidence of negative quadratic trends (i.e., inverted-U-shaped activity) in both groups.

\section{Aim 2: Neuronal Inefficiency in Schizophrenia}

Neuronal inefficiency is operationalized as one group — such as patients-needing to produce greater neuronal activity to achieve a similar level of performance as another groupsuch as controls. Thus, neuronal inefficiency should be evidenced by large group main or interaction effects in the conditional performance domain. In Figure 3, neuronal inefficiency should be evidenced by diverging patterns of neuronal activity (and also by meaningful group effect sizes for conditional performance in Supplemental Table 1).

Contrary to the neuronal inefficiency model, the patterns of activity are mostly overlapping, with the possible exception of the left aMFG. That is, as reported in Supplemental Table 1, most group and group interaction effect size parameters were small, and their confidence intervals included zero. However, there was a medium-sized group-by-quadratic interaction effect with a nonzero confidence interval in the left aMFG. Specifically, SZs showed a more negative quadratic slope. In Figure 3, it can be seen that SZs produced more peaked activity in the aMFG in comparison to HCs, who produced a more muted, flatter pattern of activity.

\section{Aim 3: Correlates of Inefficient Neuronal Activity}

To better understand the meaning of aMFG activity, we explored the demographic, cognitive, and clinical correlates of BOLD response within the ROI in comparison to activity observed within the pMFG. We operationalized "inefficient" activity as SZ BOLD signal that was in excess to the signal 
atients diagnosed with schizophrenia Healthy comparison subjects
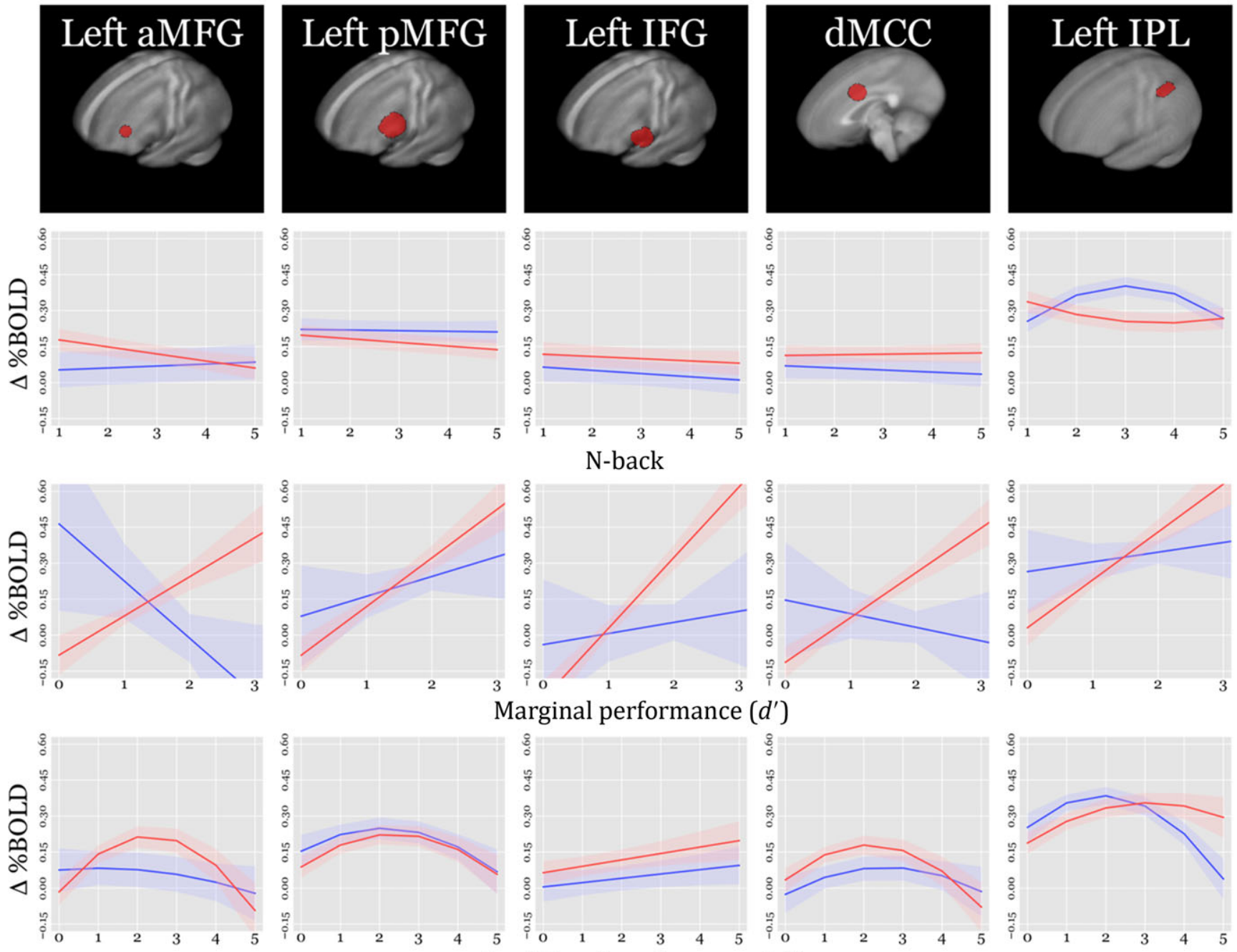

Marginal performance $\left(d^{\prime}\right)$
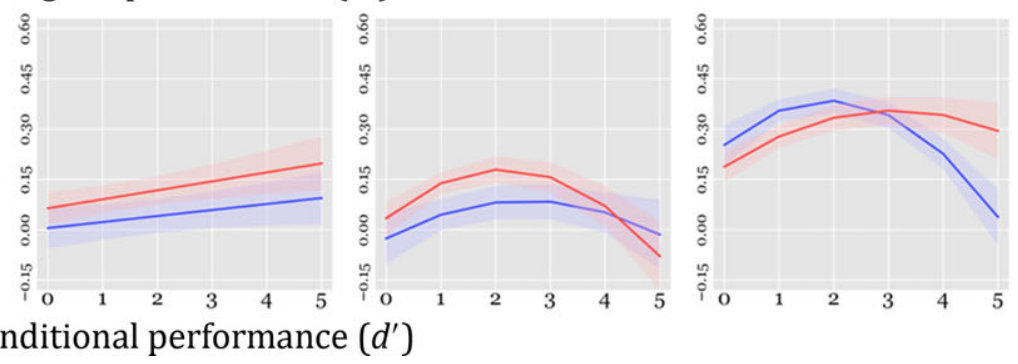

Fig. 3. Best-fitting regression model predictions and standard errors (shaded regions) for percentage change in blood-oxygenation-level-dependent (BOLD) response as a function of N-back load, marginal performance, and conditional performance. Patients diagnosed with schizophrenia and plotted in red and healthy comparison subjects are plotted in blue. ROI = region of interest; aMFG $=$ anterior middle frontal gyrus; pMFG $=$ posterior middle frontal gyrus; IFG $=$ inferior frontal gyrus; dMCC $=$ dorsal midcingulate cortex; IPL $=$ inferior parietal lobule .

observed among HCs at individually matched levels of $\mathrm{N}$ back load.

Specifically, within the conditional performance domain (bottom row of Figure 3), we noted that peak BOLD activity generally occurred between discriminability $\left(d^{\prime}\right)$ values of 23 . This is equivalent to a performance accuracy range for target items of approximately 69\%-84\% (after accounting for response bias). Matched load was defined as the N-back condition that was likely to produce discriminability $\left(d^{\prime}\right)$ values of 2.5 ( $77 \%$ performance accuracy) for each subject.

Figure 4 presents correlations (with $95 \%$ confidence intervals) of "inefficient" BOLD activity in the pMFG and aMFG with demographic, clinical, and cognitive variables. Only the group correlation (first line) includes HCs; all other correlations are based on the SZ group alone. The general descriptive pattern suggests that while greater BOLD activity was broadly associated with better health (e.g., younger, fewer symptoms, and better cognitive functioning), the pattern of association is stronger for the pMFG relative to the aMFG.

\section{Whole-Brain, Voxel-Wise Analyses}

Results of whole-brain, voxel-wise analyses indicated significant group differences in BOLD activity overall, but no significant group-by-quadratic conditional performance effects. Panel A of Figure 5 plots results from whole-brain, voxelwise analyses comparing average activity collapsed over groups across all WM load conditions to the low-level baseline. Colors indicate effect sizes presented as Cohen's $d$. Redder colors indicate greater BOLD signal relative to baseline. Bluer colors indicate less BOLD signal relative to baseline. Only voxels surviving false-positive rate corrections 

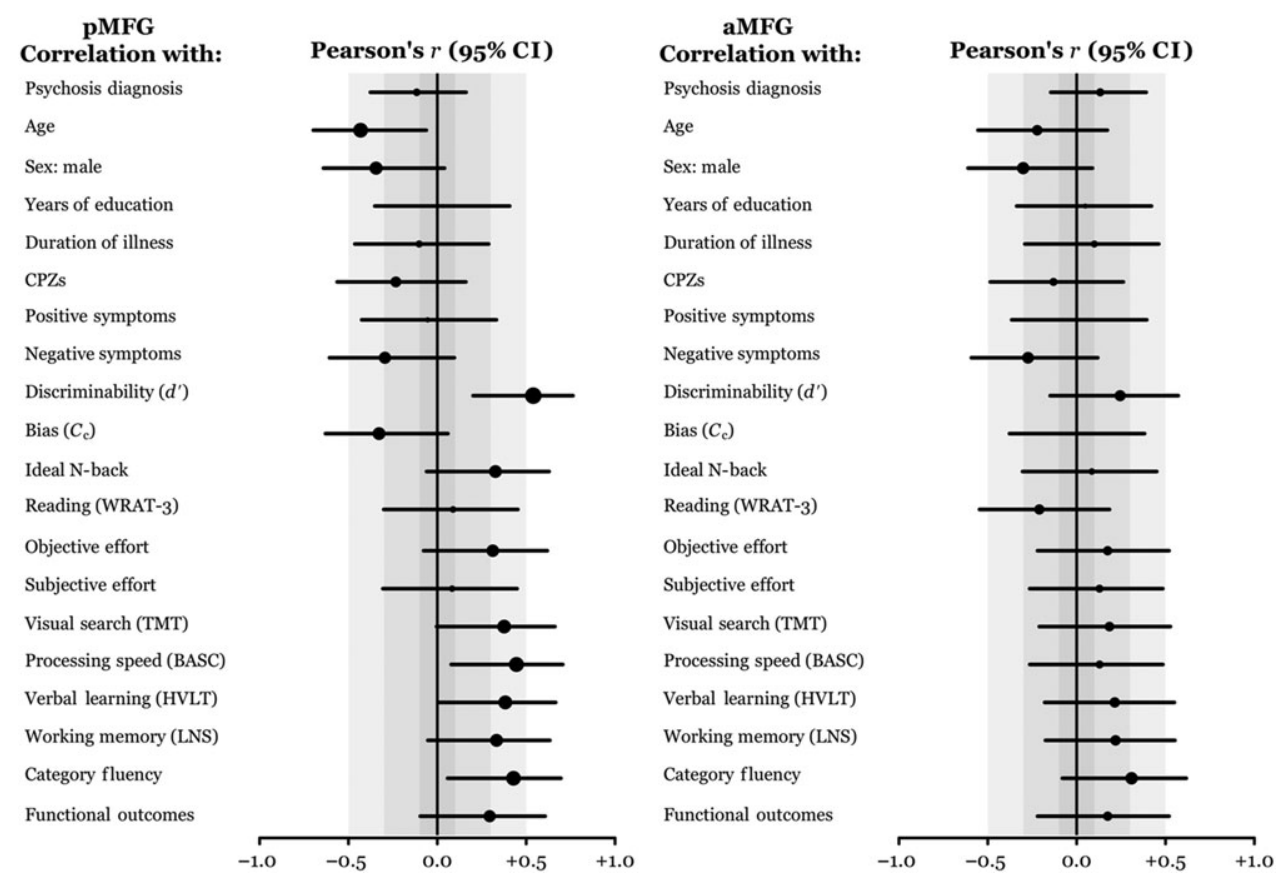

Fig. 4. Neuronal activity at ideal task difficulty. Correlations with psychosis diagnosis include healthy comparison participants and patients diagnosed with psychosis. All other correlations include only patients diagnosed with psychosis. pMFG = posterior middle frontal gyrus; $\mathrm{aMFG}=$ anterior middle frontal gyrus; $\mathrm{CPZs}=$ Chlorpromazine equivalents; WRAT-3 = Wide Range Achievement Test 3rd edition; TMT = Trial Making Test; BASC = Brief Assessment of Cognition in Schizophrenia Symbol Coding; HVLT = Hopkins Verbal Learning Test; LNS = Letter-Number Sequencing.

(ETAC) are shown in color. The results indicate bilateral increases in BOLD signal within regions generally considered to be part of the task-positive network (e.g., frontal eye fields, DLPFC and VLPFC, dMCC, and IPL). Concurrently, there are bilateral decreases in BOLD signal within regions considered to be part of the task-negative or default mode network (e.g., posterior cingulate, medial frontal gyrus, and middle temporal gyrus). Panel B of Figure 5 plots results from whole-brain, voxel-wise analyses comparing group differences. Nearly, all of the significant voxels are within default mode brain regions, with SZs showing greater activity.

\section{DISCUSSION}

We largely confirmed our hypothesis that conditional performance analysis would uniquely reveal inverted-U-shaped neuronal activity in both patients and controls. Although analyses based on task difficulty alone revealed one ROI with inverted-U-shaped neuronal activity for HCs, results suggested the oppositive pattern for SZs. These results are consistent with the "left-shift" in that the neuronal response curves for SZs appears to peak at lower levels of difficulty. Thus, differences in neuronal activity between groups as a function of load were largely determined by (or reflected in) differences in cognitive performance.

Our second aim was to explore whether patients would show greater activity in comparison to controls (i.e., neural inefficiency) for similar levels of performance. In most
ROIs, SZs demonstrated a very similar pattern of neuronal activity to HCs (bottom panel of Figure 3). Only the aMFG differed; specifically, SZs demonstrated a more peaked inverted-U-shaped load-response curve in the aMFG. Interpretively, it appears that HCs exhibited flat, modest activity in this region, and SZs exhibited highly peaked activity (i.e., both high and low activity depending on performance level).

Our third aim was to explore demographic, clinical, and cognitive correlates of inefficient neuronal activity. Perhaps surprisingly, "inefficient" BOLD activity in the aMFG appears to be a sign of relatively better health in SZs. Changes in PFC activity in both schizophrenia and aging research are sometimes interpreted as evidence of compensatory activation (Cabeza et al., 2018; Manoach et al., 1999). However, our results suggest that greater pMFG activity relative to the aMFG is a stronger marker of cognitive health in schizophrenia. Thus, whether aberrant aMFG activity should be interpreted as compensation, a deficit, or both, is unclear. Ciesklik el al. (2013) argue that that there is an anterior-ventral DLPFC that is more strongly connected to the anterior cingulate, and a posterior-dorsal DLPFC that is more strongly correlated with bilateral intraparietal sulci. Whereas the anterior-ventral DLPFC is more strongly related to attention and inhibition, the posterior network is more strongly related to action execution and WM. Interestingly, in Figure 3, it can be seen that conditional performance predicted a similarly elevated pattern of activity in the aMFG and dMCC for SZs relative to HCs. More peaked activity in the 
(a) Average activity combining groups

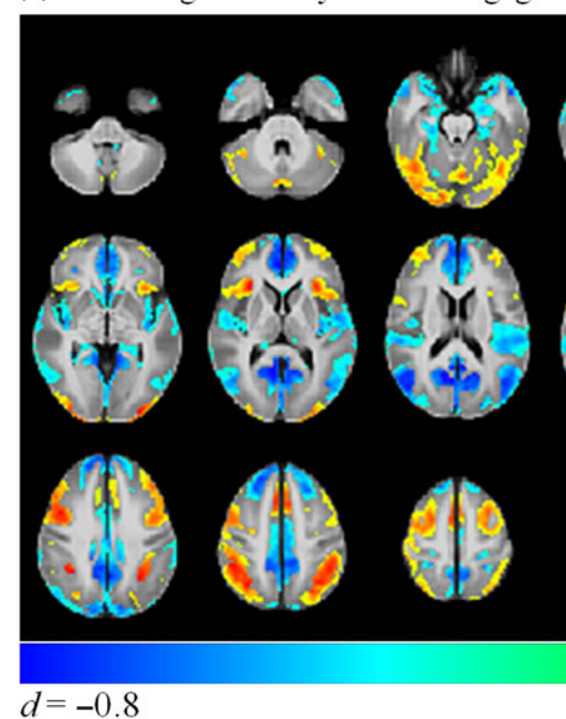

(b) Group difference in activity

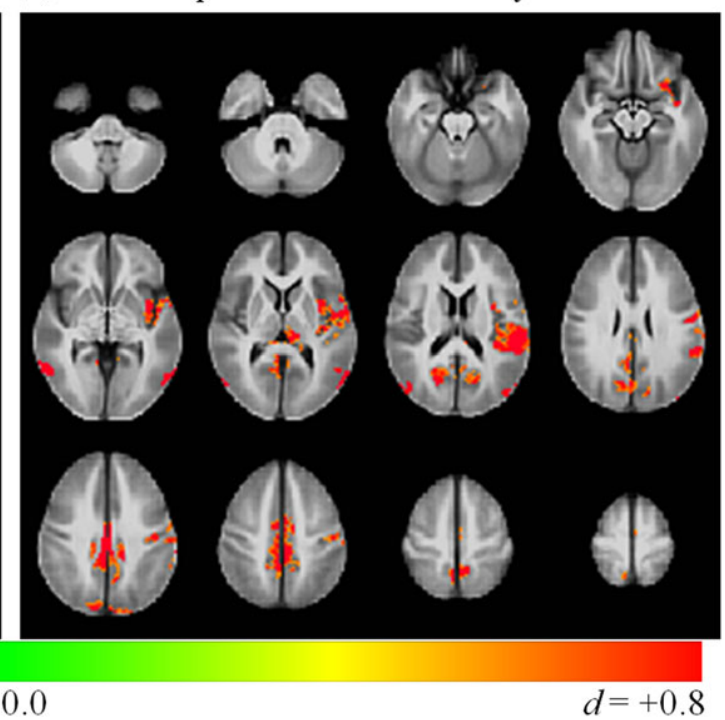

Fig. 5. (A) Results from whole-brain, voxel-wise analyses comparing average activity across all working memory load conditions to a lowlevel baseline (fixation cross). Colors indicate effect sizes presented as Cohen's $d$. Redder colors indicate relative greater BOLD signal relative to baseline. Bluer colors indicate relative lesser BOLD signal relative to baseline. Only voxels surviving false-positive rate correlation (ETAC) are shown in color. (B) Results from whole-brain, voxel-wise analyses for group differences in average activity across all working memory load conditions to a low-level baseline (fixation cross). Colors indicate effect sizes presented as Cohen's $d$. Red colors indicate relative greater BOLD signal in patients diagnosed with schizophrenia. Blue colors indicate relative lesser BOLD signal in patients diagnosed with schizophrenia. Only voxels surviving false-positive rate correlation $($ ETAC) are shown in color. Neurological orientation: left side of figure $=$ left hemisphere and right side of figure $=$ right hemisphere .

aMFG among SZs could be indicative of greater need to exert control over attention and inhibition during task engagement. Nonetheless, within patients, the suggestion from these findings is that exertion of greater cognitive control is associated with better cognitive health overall. Thus, there is at least some support for the idea of compensation. It is also possible that more diffuse activation topography reflects dedifferentiation or failure to specialize (Koen \& Rugg, 2019).

Whole-brain, voxel-wise analyses suggested that the greatest differences in neuronal activity between SZs and HCs were in activity within the task-negative or default mode network (Raichle et al., 2001). These findings support prior work suggesting that patients fail to suppress default mode network activity during task engagement, particularly among cognitively impaired individuals (Zhou et al., 2016). Notably, our results do not suggest that patients were simply disengaged with the task overall. We found no significant differences in activity within the task-positive network, including the specific ROIs targeted, and no differences in inverted-U-shaped (quadratic) activity.

\section{Limitations}

A counterargument to the proposed conditional performance analysis strategy might claim that different levels of task difficulty are qualitatively different. That is, although load manipulations are common in clinical studies, some view distinct load conditions as measures of different and unique subconstructs (Ragland et al., 2002). In previous work, we have shown that the psychometric dimensionality of the N-back task is well explained by the signal detection model (Thomas, Brown, Patt, \& Duffy, 2020). But even so, psychometric dimensionality and neuronal response dimensionality are not one and the same (Brown, Thomas, \& Patt, 2017). Shifts in strategy and learning over time are additional concerns (Juvina \& Taatgen, 2007); although these can sometimes be managed with proper counterbalancing of load conditions, changing in strategy and learning are likely to add error to brain and cognitive measures. Another concern is that our results demonstrated the use of conditional performance analysis using a single behavioral paradigm. Additional studies are needed to determine whether the utility of this approach applies to other tasks and constructs. Also, although all participants denied recent marijuana use, we did not record positive urine screens for marijuana, and thus it is possible that marijuana use could have impacted group differences in cognitive performance or neuronal activity. Finally, it should be noted that there is a growing concern about the replicability of findings from small sample studies in the fMRI literature (Turner, Paul, Miller, \& Barbey, 2018). To counter this concern in the current study, we chose a hypothesis-based ROI approach, performed multiple runs of our task, and used a cognitive paradigm with high individual differences reliability; all strategies that should improve the replicability of our findings (Nee, 2019; Poldrack, Mumford, \& Nichols, 2011). Given these choices, we ultimately had enough power to detect differences in model fit that were equivalent to an increase in variance explained of 
just .02 (i.e., small). Additionally, as shown in Supplemental Figure 2, the false discovery rate-corrected $p$ values in the current study followed a bimodal distribution, where the majority of nonsignificant values were far above the nominal cutoff of .05.

\section{Summary}

Results confirm the inverted-U-shaped theory of brain activation as a function of task difficulty. They further suggest that interpretations of neuronal activity differences between clinical and healthy comparison groups can be flawed when not considered within the contexts of individual and group differences in ability and task difficulty. While future research is needed to determine whether conditional performance analysis is applicable across cognitive domains, the results of this study — at a minimum - suggest that researchers should consider the use of conditional performance analysis to completement more traditional analysis strategies used in task-based fMRI research. The approach is particularly relevant to populations that differ in cognitive functioning. As demonstrated here, conditional performance analysis can be implemented for both ROI and whole-brain, voxel-wise analyses. Moreover, the results underscore the importance of cautious interpretation of brain activation differences between groups that differ on ability and call into question previous theories that use performance data to interpret the meaning of more or less neuronal activity in individuals with cognitive disorders.

\section{SUPPLEMENTARY MATERIAL}

To view supplementary material for this article, please visit https://doi.org/10.1017/S1355617721000424

\section{FINANCIAL SUPPORT}

Research reported in this publication was supported, in part, by the National Institute of Mental Health of the National Institutes of Health under award number K23 MH102420. The content is solely the responsibility of the authors and does not necessarily represent the official views of the National Institutes of Health.

\section{CONFLICT OF INTEREST}

The authors report no conflicts of interest related to this research.

\section{REFERENCES}

Andreasen, N.C. (1984a). The Scale for the assessment of negative symptoms (SANS). Iowa City: The University of Iowa.

Andreasen, N.C. (1984b). The Scale for the assessment of positive symptoms (SAPS). Iowa City: The University of Iowa.

Barch, D.M. \& Smith, E. (2008). The cognitive neuroscience of working memory: relevance to CNTRICS and schizophrenia.
Biological Psychiatry, 64(1), 11-17. https://doi.org/10.1016/j. biopsych.2008.03.003.

Bates, D., Mächler, M., Bolker, B., \& Walker, S. (2015). Fitting linear mixed-effects models using lme4. Journal of Statistical Software, 67(1), 1-48. https://doi.org/10.18637/jss.v067.i01.

Benjamini, Y. \& Hochberg, Y. (1995). Controlling the false discovery rate: a practical and powerful approach to multiple testing. Journal of the Royal Statistical Society. Series B (Methodological), 57(1), 289-300. JSTOR. Retrieved from JSTOR.

Boone, K.B., Salazar, X., Lu, P., Warner-Chacon, K., \& Razani, J. (2002). The Rey 15-item recognition trial: a technique to enhance sensitivity of the Rey 15-item memorization test. Journal of Clinical and Experimental Neuropsychology, 24(5), 561-573. https://doi.org/10.1076/jcen.24.5.561.1004.

Brown, G.G. \& Eyler, L.T. (2006). Methodological and conceptual issues in functional magnetic resonance imaging: applications to schizophrenia research. Annual Review of Clinical Psychology, 2, 51-81. https://doi.org/10.1146/annurev.clinpsy.2.022305.095241.

Brown, G.G., Thomas, M.L., \& Patt, V. (2017). Parametric model measurement: reframing traditional measurement ideas in neuropsychological practice and research. The Clinical Neuropsychologist, 31(6-7), 1047-1072. https://doi.org/10. 1080/13854046.2017.1334829.

Brown, G.G. \& Thompson, W.K. (2010). Functional brain imaging in schizophrenia: selected results and methods. In N.R. Swerdlow (Ed.), Behavioral neurobiology of schizophrenia and its treatment (pp. 181-214). Berlin, Heidelberg: Springer Berlin Heidelberg. https://doi.org/10.1007/7854_2010_54.

Cabeza, R., Albert, M., Belleville, S., Craik, F.I.M., Duarte, A., Grady, C.L., ... Rajah, M.N. (2018). Maintenance, reserve and compensation: the cognitive neuroscience of healthy ageing. Nature Reviews Neuroscience, 19(11), 701-710. https://doi.org/ 10.1038/s41583-018-0068-2.

Callicott, J.H. (1999). Physiological characteristics of capacity constraints in working memory as revealed by functional MRI. Cerebral Cortex, 9(1), 20-26. https://doi.org/10.1093/cercor/9. 1.20 .

Callicott, J.H., Bertolino, A., Mattay, V.S., Langheim, F.J., Duyn, J., Coppola, R., ... Weinberger, D.R. (2000). Physiological dysfunction of the dorsolateral prefrontal cortex in schizophrenia revisited. Cerebral Cortex (New York, N.Y.: 1991), 10(11), 1078-1092.

Callicott, J.H., Mattay, V.S., Verchinski, B.A., Marenco, S., Egan, M.F., \& Weinberger, D.R. (2003). Complexity of prefrontal cortical dysfunction in schizophrenia: more than up or down. American Journal of Psychiatry, 160(12), 2209-2215. https:// doi.org/10.1176/appi.ajp.160.12.2209.

Cieslik, E.C., Zilles, K., Caspers, S., Roski, C., Kellermann, T.S., Jakobs, O., ... Eickhoff, S.B. (2013). Is there "one" DLPFC in cognitive action control? Evidence for heterogeneity from co-activation-based parcellation. Cerebral Cortex, 23(11), 2677-2689. https://doi.org/10.1093/cercor/bhs256.

Cohen, J.D., Perlstein, W.M., Braver, T.S., Nystrom, L.E., Noll, D.C., Jonides, J., \& Smith, E.E. (1997). Temporal dynamics of brain activation during a working memory task. Nature, 386(6625), 604-608. https://doi.org/10.1038/386604a0.

Cox, R.W. (1996). AFNI: software for analysis and visualization of functional magnetic resonance neuroimages. Computers and Biomedical Research, 29(3), 162-173. https://doi.org/DOI10. 1006/cbmr.1996.0014. 
Cox, R.W. (2019). Equitable thresholding and clustering: a novel method for functional magnetic resonance imaging clustering in AFNI. Brain Connectivity, 9(7), 529-538. https://doi.org/10. 1089/brain.2019.0666.

Desmond, J.E., Gabrieli, J.D., Wagner, A.D., Ginier, B.L., \& Glover, G.H. (1997). Lobular patterns of cerebellar activation in verbal working-memory and finger-tapping tasks as revealed by functional MRI. The Journal of Neuroscience: The Official Journal of the Society for Neuroscience, 17(24), 9675-9685.

First, M.B., Williams, J.B.W., Karg, R.S., \& Spitzer, R.L. (2015). Structured clinical interview for DSM-5-research version (SCID-5 for DSM-5, research version; SCID-5-RV). Arlington, VA: American Psychiatric Association.

Glahn, D.C., Ragland, J.D., Abramoff, A., Barrett, J., Laird, A.R., Bearden, C.E., \& Velligan, D.I. (2005). Beyond hypofrontality: a quantitative meta-analysis of functional neuroimaging studies of working memory in schizophrenia. Human Brain Mapping, 25(1), 60-69. https://doi.org/10.1002/hbm. 20138

Glover, G.H., Li, T.Q., \& Ress, D. (2000). Image-based method for retrospective correction of physiological motion effects in fMRI: RETROICOR. Magnetic Resonance in Medicine, 44(1), 162-167. https://doi.org/10.1002/1522-2594(200007)44:1<162: aid-mrm23>3.0.co;2-e

Goldberg, T.E., Berman, K.F., Fleming, K., Ostrem, J., Van Horn, J.D., Esposito, G., ... Weinberger, D.R. (1998). Uncoupling cognitive workload and prefrontal cortical physiology: a PET rCBF study. NeuroImage, 7(4), 296-303. https://doi.org/10. 1006/nimg.1998.0338.

Goodman, S.H., Sewell, D.R., Cooley, E.L., \& Leavitt, N. (1993). Assessing levels of adaptive functioning: the role functioning scale. Community Mental Health Journal, 29(2), 119-131. https://doi.org/10.1007/BF00756338.

Green, M.F., Kern, R.S., Braff, D.L., \& Mintz, J. (2000). Neurocognitive deficits and functional outcome in schizophrenia: are we measuring the "right stuff"? Schizophrenia Bulletin, 26(1), 119-136.

Green, M.F., Nuechterlein, K.H., Gold, J.M., Barch, D.M., Cohen, J., Essock, S., . . Marder, S.R. (2004). Approaching a consensus cognitive battery for clinical trials in schizophrenia: the NIMHMATRICS conference to select cognitive domains and test criteria. Biological Psychiatry, 56(5), 301-307. https://doi.org/10. 1016/j.biopsych.2004.06.023.

Gur, R.C., Erwin, R.J., \& Gur, R.E. (1992). Neurobehavioral probes for physiologic neuroimaging studies. Archives of General Psychiatry, 49(5), 409-414. https://doi.org/10.1001/archpsyc. 1992.01820050073013.

Hart, S.G. \& Staveland, L.E. (1988). Development of NASA-TLX (Task Load Index): results of empirical and theoretical research. In Advances in psychology, 52. Human mental workload (pp. 139-183). Oxford, England: North-Holland. https://doi.org/10. 1016/S0166-4115(08)62386-9.

Hox, J.J. (2010). Multilevel analysis: techniques and applications, 2nd ed. New York, NY, US: Routledge/Taylor \& Francis Group.

Jansma, J. (2004). Working memory capacity in schizophrenia: a parametric fMRI study. Schizophrenia Research, 68(2-3), 159-171. https://doi.org/10.1016/S0920-9964(03)00127-0.

Jenkinson, M., Beckmann, C.F., Behrens, T.E., Woolrich, M.W., \& Smith, S.M. (2012). FSL. Neuroimage, 62(2), 782-790. https:// doi.org/10.1016/j.neuroimage.2011.09.015.
Juvina, I. \& Taatgen, N. (2007). Modeling control strategies in the nback task. Proceedings of the 8th International Conference on Cognitive Modeling, 73-78. https://iccm-conference.neocities. org/2007/files/juvina_taatgen.pdf.

Kalkstein, S., Hurford, I., \& Gur, R.C. (2010). Neurocognition in schizophrenia. Current Topics in Behavioral Neurosciences, 4, 373-390. https://doi.org/10.1007/7854_2010_42.

Karlsgodt, K.H., Sanz, J., van Erp, T.G.M., Bearden, C.E., Nuechterlein, K.H., \& Cannon, T.D. (2009). Re-evaluating dorsolateral prefrontal cortex activation during working memory in schizophrenia. Schizophrenia Research, 108(1-3), 143-150. https://doi.org/10.1016/j.schres.2008.12.025.

Koen, J.D. \& Rugg, M.D. (2019). Neural dedifferentiation in the aging grain. Trends in Cognitive Sciences, 23(7), 547-559. https://doi.org/10.1016/j.tics.2019.04.012.

Lee, J. \& Park, S. (2005). Working memory impairments in schizophrenia: a meta-analysis. Journal of Abnormal Psychology, 114(4), 599-611. https://doi.org/10.1037/0021-843X.114.4.599.

Manoach, D.S. (2003). Prefrontal cortex dysfunction during working memory performance in schizophrenia: reconciling discrepant findings. Schizophrenia Research, 60(2-3), 285-298. https://doi. org/10.1016/S0920-9964(02)00294-3.

Manoach, D.S., Gollub, R.L., Benson, E.S., Searl, M.M., Goff, D.C., Halpern, E., . . R Rauch, S.L. (2000). Schizophrenic subjects show aberrant fMRI activation of dorsolateral prefrontal cortex and basal ganglia during working memory performance. Biological Psychiatry, 48(2), 99-109. https://doi.org/10.1016/ s0006-3223(00)00227-4.

Manoach, D.S., Press, D.Z., Thangaraj, V., Searl, M.M., Goff, D.C., Halpern, E., ... Warach, S. (1999). Schizophrenic subjects activate dorsolateral prefrontal cortex during a working memory task, as measured by fMRI. Biological Psychiatry, 45(9), 1128-1137. https://doi.org/10.1016/S0006-3223(98)00318-7.

Manoach, D.S., Schlaug, G., Siewert, B., Darby, D.G., Bly, B.M., Benfield, A., ... Warach, S. (1997). Prefrontal cortex fMRI signal changes are correlated with working memory load. Neuroreport, 8(2), 545-549.

Miyake, A. \& Shah, P. (1999). Toward unified theories of working memory: emerging general consensus, unresolved theoretical issues, and future research directions. In M. Akira, S. Priti (Eds.), Models of working memory: mechanisms of active maintenance and executive control (pp. 442-481). Cambridge, UK: Cambridge University Press.

Nee, D.E. (2019). FMRI replicability depends upon sufficient individual-level data. Communications Biology, 2(1), 1-4. https://doi. org/10.1038/s42003-019-0378-6.

Owen, A.M., McMillan, K.M., Laird, A.R., \& Bullmore, E. (2005). N-back working memory paradigm: a meta-analysis of normative functional neuroimaging studies. Human Brain Mapping, 25(1), 46-59. https://doi.org/10.1002/hbm.20131.

Peirce, J., Gray, J.R., Simpson, S., MacAskill, M., Höchenberger, R., Sogo, H., ... Lindeløv, J.K. (2019). PsychoPy2: experiments in behavior made easy. Behavior Research Methods, 51(1), 195-203. https://doi.org/10.3758/s13428-018-01193-y.

Poldrack, R.A., Mumford, J.A., \& Nichols, T.E. (2011). Handbook of functional MRI data analysis, 1st ed. Cambridge, New York, Melbourne, Madrid: Cambridge University Press.

Potkin, S.G., Turner, J.A., Brown, G.G., McCarthy, G., Greve, D.N., Glover, G.H., ... FBIRN. (2009). Working memory and DLPFC inefficiency in schizophrenia: the FBIRN study. Schizophrenia Bulletin, 35(1), 19-31. https://doi.org/10.1093/ schbul/sbn162. 
Ragland, J.D., Turetsky, B.I., Gur, R.C., Gunning-Dixon, F., Turner, T., Schroeder, L., ... Gur, R.E. (2002). Working memory for complex figures: an fMRI comparison of letter and fractal n-back tasks. Neuropsychology, 16(3), 370-379.

Raichle, M.E., MacLeod, A.M., Snyder, A.Z., Powers, W.J., Gusnard, D.A., \& Shulman, G.L. (2001). A default mode of brain function. Proceedings of the National Academy of Sciences of the United States of America, 98(2), 676-682. https://doi.org/10. 1073/pnas.98.2.676.

Rex, D.E., Ma, J.Q., \& Toga, A.W. (2003). The LONI pipeline processing environment. Neuroimage, 19(3), 1033-1048. https://doi.org/10.1016/S1053-8119(03)00185-X.

Thomas, M.L., Brown, G.G., Gur, R.C., Moore, T.M., Patt, V.M., Risbrough, V.B., \& Baker, D.G. (2018). A signal detectionitem response theory model for evaluating neuropsychological measures. Journal of Clinical and Experimental Neuropsychology, 40(8), 745-760. https://doi.org/10.1080/ 13803395.2018.1427699.

Thomas, M.L., Brown, G.G., Patt, V.M., \& Duffy, J.R. (2020). Latent variable modeling and adaptive testing for experimental cognitive psychopathology research. Educational and Psychological Measurement, 001316442091989. https://doi. org/10.1177/0013164420919898.

Turner, B.O., Paul, E.J., Miller, M.B., \& Barbey, A.K. (2018). Small sample sizes reduce the replicability of task-based fMRI studies.
Communications Biology, 1(1), 1-10. https://doi.org/10.1038/ s42003-018-0073-z.

Van Snellenberg, J.X., Girgis, R.R., Horga, G., van de Giessen, E., Slifstein, M., Ojeil, N., ... Abi-Dargham, A. (2016). Mechanisms of working memory impairment in schizophrenia. Biological Psychiatry, 80(8), 617-626. https://doi.org/10.1016/ j.biopsych.2016.02.017.

Webb, J.R., Schroeder, M.I., Chee, C., Dial, D., Hana, R., Jefee, H., ... Molitor, P. (2013). Left-handedness among a community sample of psychiatric outpatients suffering from mood and psychotic disorders. SAGE Open, 3(4), 215824401350316. https:// doi.org/10.1177/2158244013503166.

Westbrook, A. \& Braver, T.S. (2015). Cognitive effort: a neuroeconomic approach. Cognitive, Affective \& Behavioral Neuroscience, 15(2), 395-415. https://doi.org/10.3758/s13415-015-0334-y.

Zhou, L., Pu, W., Wang, J., Liu, H., Wu, G., Liu, C., ... Liu, Z. (2016). Inefficient DMN suppression in schizophrenia patients with impaired cognitive function but not patients with preserved cognitive function. Scientific Reports, 6(1), 21657. https://doi. org/10.1038/srep21657.

Zou, Q., Gu, H., Wang, D.J.J., Gao, J.-H., \& Yang, Y. (2011). Quantification of load dependent brain activity in parametric $\mathrm{N}$-Back working memory tasks using pseudo-continuous arterial spin labeling (pCASL) perfusion imaging. Journal of Cognitive Science, 12(2), 127-210. 